\title{
From Chern-Simon, Holography and Scale Relativity to Dark Energy
}

\author{
Mohamed S. El Naschie \\ Department of Physics, Faculty of Science, University of Alexandria, Alexandria, Egypt \\ Email: Chaossf@aol.com
}

Received 14 March 2014; revised 14 April 2014; accepted 21 April 2014

Copyright (C) 2014 by author and Scientific Research Publishing Inc.

This work is licensed under the Creative Commons Attribution International License (CC BY).

http://creativecommons.org/licenses/by/4.0/

(c) (i) Open Access

\begin{abstract}
Chern-Simon theory and the holographic principle as well as scale relativity are used to find out the exact value of cosmic ordinary and dark energy density. The result agrees completely with previously obtained ones as well as with accurate cosmic measurements.
\end{abstract}

\section{Keywords}

Holographic Boundary, Chern-Simon Theory, Dark Energy, Cosmic Expansion, Fractal-Cantorian Spacetime

\section{Introduction and Background Information}

We start the present analysis of determining the dark energy density of the cosmos [1]-[12] in a reverse order to the heading of the paper at hand by recalling an important result of Nottale's scale relativity [11], namely that the inverse coupling of GUT tends to a "highly particular" value $\bar{\alpha}_{u}(\mathrm{GUT})=4 \pi^{2}$ as the energy tends to infinity [13]. This is stated explicitly in his well-known book [13] on page 264. In other words the relevant running of $\mathrm{U}(1), \mathrm{SU}(2)$ and $\mathrm{SU}(3)$ does not intersect in the conventional way but rather tends asymptotically towards an imaginary intersection point at infinity. This is of course a consequence of the fractal nature of Nottale's theory and in that respect quite satisfying and logically appealing as far as our Cantorian view point is concerned [4][13]. However we showed on an earlier occasion [14] [15] that continuity introduces spurious terms to the relevant equations and transfiniteness require higher order correction terms [15] similar in spirit to radiation corRections albeit in this case Cantorian-fractal corrections leading to $4 \rightarrow 4+\phi^{3}$ and $\pi^{2} \rightarrow 10$ where $\phi=(\sqrt{5}-1) / 2$ [16]. The exact transfinite inverse coupling in this case is thus not $4 \pi^{2}=39.47$ but rather ( $4+$ $\left.\phi^{3}\right)(10)=42+2 k=42.36067977$ [17]. The interesting point in this slight discrepancy of the magnitude of $\bar{\alpha}_{u}$ 
comes to the fore when we consider the automorphism of the classical 336 degrees of freedom Klein modular curve, then we have 336/2 = 168 such automorphism [18]. Again looking at a relevant cycle of this curve which plays the role of a holographic boundary to a bulk given by E8E8 exceptional Lie symmetry group, then we arrive at what we may call field strength of the magnitude $F=168 / 2=84$ [19]. This gives us our first result of interest, namely that

$$
F=2 \bar{\alpha}_{u}=(2)(42)=84 .
$$

The exact transfinitely corrected expression is of course [16]

$$
F_{c}=(2)(42+2 k)=84+4 k=84.72135954
$$

where $k=\phi^{5}\left(1-\phi^{5}\right)$ and $\phi=(\sqrt{5}-1) / 2=0.618033989$. On the other hand field strength $F$ corresponds to the curvature in the Chern-Simon theory of fiber bundles [20] and is given by $F=8 \pi^{2}$. In other words it harmonizes with scale relativity when written in the form

$$
F_{c}=2 \bar{\alpha}_{u}=(2)\left(4 \pi^{2}\right)=8 \pi^{2} .
$$

In the next section of the present work we will examine the preceding result in some detail [20]-[44].

\section{Field Strength and Dark Energy}

Our present thesis however is that $F=2 \bar{\alpha}_{u}=(2)(42)=84$ is the correct expression and that it leads us in this way to a correct appreciation of the exact value and the role of the holographic degrees of freedom in determining the ratio between the directly accessible to the indirectly accessible energy stored in our fiber bundle-like universe and in fact a superstring conception of our cosmos [9]-[12] [21]-[25]. Our point is that $F=84$ minus 10 give us the dark energy component of the total energy density as expressed by the sum of all the fundamental inverse couplings [16] [21].

$$
\sum_{n=1}^{n=4} \bar{\alpha}_{i}=\bar{\alpha}_{1}+\bar{\alpha}_{2}+\bar{\alpha}_{3}+\bar{\alpha}_{4}=\bar{\alpha}_{1}+\bar{\alpha}_{2}+\left(\bar{\alpha}_{3,1}+\bar{\alpha}_{3,2}\right)+\bar{\alpha}_{4}=60+30+(8+1)+1=100
$$

That means

$$
\bar{\alpha}(\text { Dark })=F-\left(\bar{\alpha}_{3}+\bar{\alpha}_{4}\right)=84-10=74 .
$$

In other words 74 percent of the total energy is pure dark energy not counting dark matter. Our sum may therefore be rewritten as

$$
\sum_{1}^{4} \bar{\alpha}_{i}=22+4+74=100
$$

where the ordinary energy component is given by $\bar{\alpha}$ (ordinary) $=4$ and the dark matter component is given by $\bar{\alpha}$ (dark matter) $=22$. Needless to say, the preceding analysis and conclusions need further elaboration for a proper understanding of the deep message we are trying to convey. To start with the normed hundred percent sum of all the fundamental inverse coupling needs refinement and elucidation. The $\bar{\alpha}_{1}, \bar{\alpha}_{2}$ and $\bar{\alpha}_{3}$ are idealized theoretical values which happen to be related to the electroweak scale. They are very close indeed to the experimental values found in numerous experiments [26] [27] to be $\bar{\alpha}_{1} \simeq 59, \bar{\alpha}_{2}=29, \bar{\alpha}_{3} \simeq 8$ [29] [30]. On the other hand $\bar{\alpha}_{3,2}=\bar{\alpha}_{4}=\bar{\alpha}_{Q G}=1$ are related to the quantum gravity Planck scale [26]-[29]. Never the less all the four inverse couplings enter into a special form of an exact renormalization equation from which the fundamental electromagnetic inverse constant may be reconstructed as [29] [30]

$$
\begin{aligned}
\bar{\alpha}_{o} & =\left(\bar{\alpha}_{1}\right)(1 / \phi)+\left[\bar{\alpha}_{2}=\left(\bar{\alpha}_{1} / 2\right)\right]+\bar{\alpha}_{3}+\bar{\alpha}_{4}=(60)(1 / \phi)+30+9+1 \\
& =\left(97+k_{o}\right)+40=137+k_{o}=137+\phi^{5}\left(1-\phi^{5}\right)=137.082039325
\end{aligned} .
$$

For the above reasons we regard the sum of $\bar{\alpha}_{i}$ being 100 as a weighted number of the involved space and internal dimensions just as $1 / \bar{\alpha}_{o}$ could be regarded as a cross-section or a quantum probability for a photon to capture or emit an electron while at the same time $\bar{\alpha}_{o}=137.0820393$ may be regarded as a dimension of a 
fractal space in which the electron "moves" [16] [30]. Thus 100 is a weighted total dimension comparable with the 496 of E8E8 or the 528 of the manifold of killings vector fields associated with Witten's 5D Branes in eleven dimensions [31]

$$
N_{K}^{(32)}=\left(\begin{array}{c}
11 \\
1
\end{array}\right)+\left(\begin{array}{c}
11 \\
2
\end{array}\right)+\left(\begin{array}{c}
11 \\
5
\end{array}\right)=11+55+462=(n)(n+1) / 2=(32)(32+1) / 2=528 .
$$

In this sense the subdivision of the sum of $\bar{\alpha}_{i}$ has to be undertaken more delicately to reveal its hidden inner fine structure. To start with the 22 of the sum $22+4+74$ must be $22+k=22.18033989$ to bring it in harmony with the Hardy's quantum entanglement $\phi^{5}$ and the previous result of ordinary energy

$E=\left(\phi^{5} / 2\right) m c^{2}=m c^{2} /(22+k)$. Similarly the 4 must become the ordinary energy percentage which is

$(100) /[1 /(22+k)]=4.50849187$. Consequently the pure dark energy exact percentage must be

$100-[(22+k)+(4.50849187)]=73.31116292$.

\section{Dark Energy from the Holographic Boundary}

Now we can return to the holographic theory and how to calculate the ordinary energy component directly and subsequently the dark energy and dark matter components indirectly [4]-[12] [32]-[35]. For clarity we start with the integer approximate form of the theory. We know that the holographic boundary has no gravity degrees of freedom since gravity resides inside the Bulk. Thus the only degrees of freedom are those given by $|\operatorname{SL}(2,7)|=7\left(7^{2}-1\right)=(7)(48)=336$ which is in a one to one correspondence with the degrees of freedom of Klein's modular curve as indicated earlier on. Einstein's gravity on the other hand has in $D=4$ twenty independent Riemannian components corresponding to 20 degrees of freedom in super symmetric $D=8$ space for pure gravity. From these twenty, four dimensions were already used in Einstein's spacetime so that we are left with effectively only $20-4=16$ degrees of freedom. Our reduced Lorentzian factor is now clearly equal to the ratio of these 16 to the 336 holographic degrees of freedom after amplification by adding the 20 of the Riemann-Einstein independent "degrees of freedom" and subtracting the $D^{(4)}=4$. That way we find

$$
\gamma=\frac{R^{(4)}-D^{(4)}}{|\operatorname{SL}(2,3)|+R^{(4)}-D^{(4)}}=\frac{20-4}{336+20-4}=\frac{16}{336+16}=\frac{16}{352}=\frac{1}{22}
$$

exactly as anticipated [32]-[35]. The dark energy density is consequently the maximal energy density $E=m c^{2}$ minus the ordinary energy density $E(0)=m c^{2} / 22$ which means [35]-[38]

$$
E(D)=m c^{2}(21 / 22) \text {. }
$$

For the sake of completeness we give without much elaboration two exact transfinite formulas for $E(0)$ and $E(D)$ which effectively boils down to using the transfinitely compactified holographic boundary $336 \rightarrow 336-$ 16k where $k=\phi^{5}\left(1-\phi^{5}\right)=0.18033989$ and $\phi^{5}$ is Hardy's probability of quantum entanglement [36]. Proceeding in this way one finds that

$$
E(0)=m c^{2} \frac{20-4}{336+16 k+20-4}=m c^{2} \frac{16}{356+16 k}=m c^{2} \frac{1}{22.18033989}=\left(\phi^{5} / 2\right) m c^{2} \simeq m c^{2} / 22
$$

and

$$
E(D)=1-E(0)=\left(5 \phi^{5} / 2\right) m c^{2} \simeq m c^{2}(21 / 22) .
$$

Again this is in full agreement with previous analysis [4]-[12] [32]-[38] and accurate cosmological measurements [1]-[3].

\section{Conclusion}

The present analysis of the dark and ordinary energy density in the cosmos centres is around three main ideas, namely scale relativity, Chern-Simon theory and the holographic principle. The so obtained results, i.e. $E(0)=$ 
$m c^{2} / 22$ and $E(D)=1-E(0)=m c^{2}(21 / 22)$ agree completely with all previous analysis [4]-[12] [32]-[35] as well as cosmic measurements [1]-[3]. Wider aspects of the present theory which go beyond mainstream physics and cosmology are discussed in reference [39] as well as [40] and [41] where the question of infinitely many dimensions is considered. In addition we recently made considerable advances in using the physical concepts of fluid mechanics and nonlocal elasticity to understand the nature of dark energy [41] [42]. Finally it is important to mention a major finding regarding ' $t$ Hooft's dimensional regularization and a corresponding fractal Cantorian spacetime leading to a dark energy density equal to that found using accurate cosmic measurements [43] [44].

\section{References}

[1] Linder, E.V. (2007) Resource Letter: Dark Energy and Accelerating Universe. arXiv: 0705.4102V1[astroph].

[2] Perlmutter, S. and Schmidt, B. (2003) Measuring Cosmology with Supernova. arXiv: astroph/0303428V1.

[3] Linder, E.V. (2010) Einstein's Other Gravity and the Acceleration of the Universe. arXiv: 1005.3039V2[astro-hy.co].

[4] El Naschie, M.S. (2013) A Resolution of Cosmic Dark Energy via a Quantum Entanglement Relativity Theory. Journal of Quantum Information Science, 3, 23-26.

[5] El Naschie, M.S. (2013) Quantum Gravity and Dark Energy via a New Planck Scale. Fractal Spacetime and Noncommutative Geometry in Quantum and High Energy Physics, 3, 106-119.

[6] El Naschie, M.S. (2014) Dark Energy via a Quantum Field Theory in Curved Spacetime. Journal of Modern Physics and Applications, 1, 1-7.

[7] El Naschie, M.S. (2013) From Yang-Mills Photon in Curved Spacetime to Dark Energy Density. Journal of Quantum Information Science, 3, 121-126.

[8] Marek-Crnjac, L., et al. (2013) Chaotic Fractal Tiling for the Missing Dark Energy and Veneziano Model. Applied Mathematics, 4, 22-29.

[9] El Naschie, M.S. (2013) A Rindler-KAM Spacetime Geometry and Scaling the Planck Scale Solves Quantum relativity and Explains Dark Energy. International Journal of Astronomy and Astrophysics, 3, 483-493.

[10] El Naschie, M.S. (2013) Experimentally Based Theoretical Arguments that Unruh’s Temperature, Hawking’s Vacuum Fluctuation and Rindler's Wedge Are Physically Real. American Journal of Modern Physics, 2, 357-361.

[11] Marek-Crnjac, L. and He, J.-H. (2013) An Invitation to El Naschie’s Theory of Cantorian Spacetime and Dark Energy. International Journal of Astronomy and Astrophysics, 3, 464-471.

[12] Marek-Crnjac, L. and El Naschie, M.S. (2013) Quantum Gravity and Dark Energy Using Fractal Planck Scaling. Journal of Modern Physics, 4, 31-38.

[13] Nottale, L. (1998) Fractal Spacetime and Microphysics. Towards a Theory of Scale Relativity. World Scientific, Singapore.

[14] El Naschie, M.S. (2008) Removing Spurious Non-Linearity in the Structure of Micro-Spacetime and Quantum Field Renormalization. Chaos, Solitons \& Fractals, 37, 60-64.

[15] El Naschie, M.S. (2008) Mathematical Foundations of E-Infinity via Coxeter and Reflection Groups. Chaos, Solitons \& Fractals, 37, 1267-1268.

[16] El Naschie, M.S. (2004) A Review of E-Infinity and the Mass Spectrum of High Energy Particle Physics. Chaos, Solitons \& Fractals, 19, 209-236. http://dx.doi.org/10.1016/S0960-0779(03)00278-9

[17] El Naschie, M.S. (2000) Scale Relativity in Cantorian E-Infinity Space-Time. Chaos, Solitons \& Fractals, 11, 23912395. http://dx.doi.org/10.1016/S0960-0779(99)00209-X

[18] El Naschie, M.S. (2003) Modular Groups in Cantorian E-Infinity High-Energy Physics. Chaos, Solitons \& Fractals, 16, 353-366. http://dx.doi.org/10.1016/S0960-0779(02)00440-X

[19] El Naschie, M.S. (2008) Notes on Exceptional Lie Symmetry Groups Hierarchy and Possible Implications for E-Infinity High Energy Physics. Chaos, Solitons \& Fractals, 35, 69-70.

[20] El Naschie, M.S. (2008) Fuzzy Multi-Instanton Knots in the Fabric of Space-Time and Dirac’s Vacuum Fluctuation. Chaos, Solitons \& Fractals, 38, 1260-1268. http://dx.doi.org/10.1016/j.chaos.2008.07.010

[21] El Naschie, M.S., He, J.H., Nada, S., Marek-Crnjac, L. and Helal, M.A. (2012) Golden Mean Computer for High Energy Physics. Fractal Spacetime and Noncommutative Geometry in High Energy Physics, 2, 80-92.

[22] He, J.H. (2005) Transfinite Physics. A Collection of Publications on E-Infinity Cantorian Spacetime Theory. China Scientific \& Cultural Publishing, Beijing. 
[23] Duff, M.J. (1999) The World in Eleven Dimensions. Institute of Physics Publications, Bristol.

[24] Witten, E. (1998) $2+1$ Dimensional Gravity as an Exactly Soluble System. Nuclear Physics B, 311, 46-78. http://dx.doi.org/10.1016/0550-3213(88)90143-5

[25] Kutasov, D. and Seiberg, N. (1991) Number of Degrees of Freedom, Density of States and Tachyons in String Theory and CFT. Nuclear Physics B, 358, 600-618. http://dx.doi.org/10.1016/0550-3213(91)90426-X

[26] El Naschie, M.S., et al. (2012) On the Need for Fractal Logic in High Energy Quantum Physics. International Journal of Modern Nonlinear Theory and Application, 1, 84-92. http://dx.doi.org/10.4236/ijmnta.2012.13012

[27] El Naschie, M.S. (2008) Extended Renormalization Group Analysis for Quantum Gravity and Newton's Gravitation Constant. Chaos, Solitons \& Fractals, 35, 425-431. http://dx.doi.org/10.1016/j.chaos.2007.07.059

[28] El Naschie, M.S. (2008) Transfinite Harmonization by Taking the Dissonance out of the Quantum Field Symphony. Chaos, Solitons \& Fractals, 36, 781-786. http://dx.doi.org/10.1016/j.chaos.2007.09.018

[29] El Naschie, M.S. (2008) An Outline for a Quantum Golden Field Theory. Chaos, Solitons \& Fractals, 37, 317-323. http://dx.doi.org/10.1016/j.chaos.2007.09.092

[30] El Naschie, M.S. (2009) The Theory of Cantorian Spacetime and High Energy Particle Physics (an Informal Review). Chaos, Solitons \& Fractals, 41, 2635-2646. http://dx.doi.org/10.1016/j.chaos.2008.09.059

[31] Kaku, M. (1999) Introduction to Superstrings and M-Theory. Springer, New York. http://dx.doi.org/10.1007/978-1-4612-0543-2

[32] El Naschie, M.S. (2013) Topological-Geometrical and Physical Interpretation of the Dark Energy of the Cosmos as a “Halo” Energy of the Schrödinger Quantum Wave. Journal of Modern Physics, 4, 591-596. http://dx.doi.org/10.4236/jmp.2013.45084

[33] El Naschie, M.S. (2013) Dark Energy from Kaluza-Klein Spacetime and Noether’s Theorem via Lagrangian Multiplier Method. Journal of Modern Physics, 4, 757-760. http://dx.doi.org/10.4236/jmp.2013.46103

[34] El Naschie, M.S. (2013) A Unified Newtonian-Relativistic Quantum Resolution of the Supposedly Missing Dark Energy of the Cosmos and the Constancy of the Speed of Light. International Journal of Modern Nonlinear Theory and Application, 2, 43-54. http://dx.doi.org/10.4236/ijmnta.2013.21005

[35] El Naschie, M.S. (2013) Nash Embedding of Witten’s M-Theory and the Hawking-Hartle Quantum Wave of Dark Energy. Journal of Modern Physics, 4, 1417-1428. http://dx.doi.org/10.4236/jmp.2013.410170

[36] Helal, M.A., Marek-Crnjac, L. and He, J.H. (2013) The Three Page Guide to the Most Important Results of M.S. El Naschie’s Research in E-Infinity and Quantum Physics and Cosmology. Open Journal of Microphysics, 3, 141-145. http://dx.doi.org/10.4236/ojm.2013.34020

[37] El Naschie, M.S. (2011) The Fractal Geometric Origin of Quantum Mechanics from Hardy’s Quantum Entanglement. International Journal of E-Infinity and Complexity Theory in High Energy Physics and Engineering, 1, 1-7.

[38] Marek-Crnjac, L. (2013) Cantorian Space-Time Theory-The Physics of Empty Sets in Connection with Quantum Entanglement and Dark Energy. Lambert Academic Publishing, Saarbrücken.

[39] Christianto, V. (2003) The Cantorian Super Fluid Vortex Hypothesis. Apeiron, 10, 231-248.

[40] Rubakov, V.A. (2001) Large and Infinite Extra Dimensions. Physics-Uspekhi, 44, 871-893. http://dx.doi.org/10.1070/PU2001v044n09ABEH001000

[41] El Naschie, M.S. (2014) Capillary Surface Energy Elucidation of the Cosmic Dark Energy_Ordinary Energy Duality. Open Journal of Fluid Dynamics, 4, 15-17. http://dx.doi.org/10.4236/ojfd.2014.41002

[42] El Naschie, M.S. (2014) Pinched Material Einstein Space-Time Produces Accelerated Cosmic Expansion. International Journal of Astronomy and Astrophysics, 4, 80-90. http://dx.doi.org/10.4236/ijaa.2014.41009

[43] El Naschie, M.S. (2014) Logarithmic Running of 't Hooft-Polyakov Monopole to Dark Energy. International Journal of High Energy Physics, 1, 1-5.

[44] El Naschie, M.S. (2014) Cosmic Dark Energy from ’t Hooft’s Dimensional Regularization and Witten's Topological Quantum Field Pure Gravity. Journal of Quantum Information Science, in Press. 